\title{
DYNAMIC BEHAVIOR OF TWO ELASTICALLY CONNECTED NANOBEAMS UNDER A WHITE NOISE PROCESS
}

\author{
Ivan R. Pavlović ${ }^{1}$, Ratko Pavlović ${ }^{1}$, Goran Janevski ${ }^{1}$, \\ Nikola Despenić ${ }^{1}$, Vladimir Pajković ${ }^{2}$ \\ ${ }^{1}$ Faculty of Mechanical Engineering, University of Niš, Serbia \\ ${ }^{2}$ Faculty of Mechanical Engineering, University of Montenegro, Podgorica, Montenegro
}

\begin{abstract}
This paper investigates the almost-sure and moment stability of a double nanobeam system under stochastic compressive axial loading. By means of the Lyapunov exponent and the moment Lyapunov exponent method the stochastic stability of the nano system is analyzed for different system parameters under an axial load modeled as a wideband white noise process. The method of regular perturbation is used to determine the explicit asymptotic expressions for these exponents in the presence of small intensity noises.
\end{abstract}

Key Words: Double Nanobeam, Lyapunov Exponent, Moment Lyapunov exponent, Regular Perturbation Method, White Noise Process

\section{INTRODUCTION}

Continuum modeling of nanorods, nanobeams and nanoplates has attracted attention due to its simplicity and computational efficiency. Recently, they have been extensively utilized as nanostructure components for nanoelectromechanical and microelectromechanical systems.

Based on the theory of Eringen, nanostructures have been widely studied by many researchers. The bending analysis of embedded nanoplates based on the integral formulation of the Eringen's nonlocal theory is presented in [1]. Zhang et al. [2] developed a Hencky barnet model for circular and annular single layer graphene sheets and calculated real buckling loads of such sheets with clamped and simply supported restraints.

Pavlović et al. [3] investigated the dynamic instability of coupled nanobeams. By using the direct Lyapunov method, bounds of the almost-sure asymptotic instability of a

Received April 15, 2019 / Accepted December 10, 2019

Corresponding author: Ivan R. Pavlović

Faculty of Mechanical Engineering, University of Niš, Aleksandra Medvedeva 14, 18000 Niš, Serbia

E-mail: pivan@masfak.ni.ac.rs 
coupled nanobeam system were determined as a function of different system parameters. An accurate and analytical method for investigating the dynamic instability of nanobeams based on the nonlocal continuum mechanics is given in [4]. Using the same beam theory, Jena and Chakraverty [5] investigates free vibration of nanobeams by applying the semi analytical-numerical technique called differential transform method (DTM). The effects of the compressive axial load on the properties of forced transverse vibrations of a double-beam system were investigated by Zhang et al. [6].

A systematic study of the moment Lyapunov exponents is presented in the works of Xie $[7,8]$. The method of regular perturbation was applied to obtain weak noise expansions of the moment Lyapunov exponent. By using the moment Lyapunov exponents, bounds of the almost-sure stability were determined for bounded noise [7] and real noise [8] parametric excitation. Kozić et al. [9] investigated the Lyapunov exponent and the moment Lyapunov exponents of two degrees-of-freedom linear systems subjected to white noise parametric excitation. The results from this study were further used for the study of the almost-sure and moment stability of a double-beam system under stochastic compressive axial loading. Similarly, the moment Lyapunov exponents of the stochastic parametrical Hill's equation were investigated by the same authors in [10]. Pavlović et al. [11] used this method to compare the analytically obtained results of the moment Lyapunov exponent for a simple nanobeam numerically determined for the same system. Within the concept of the Lyapunov exponent, by using the stochastic averaging method, the dynamic stability of a viscoelastic double-beam system under parametric excitations was investigated in [12].

The effect of the van der Waals forces on axial buckling of a double-walled carbon nanotube was presented in [13], where an elastic model was used to study infinitesimal buckling of a double-walled carbon nanotube under axial compression.

The present paper investigates the almost-sure stability of a double nanobeam. In Section 2 the governing differential equations of transverse motion of two elastically connected nanobeams are presented. Using the Galerkin method the system is discretizated in Section 3 , where the analyzed system is reduced to two ordinary differential equations representing only the time varying part of the solution. For the obtained discretizated form, the appropriate analytical expression of the moment Lyapunov exponent is given in Section 4. The Lyapunov exponents are then obtained using the relationship between the moment Lyapunov exponents and the Lyapunov exponent. In Section 5 numerical results and stability analysis for different system parameters are presented along with an appropriate conclusion. Concluding remarks are given in Section 6.

\section{ANALYZED SYSTEM}

Following the Eringen's nonlocal elasticity theory [14] and the Euler-Bernoulli beam theory, the partial differential equations of the two elastically connected nanobeams are given by [3]

$$
\begin{aligned}
& \mathcal{L}\left[\rho_{1} A_{1} \frac{\partial^{2} W_{1}}{\partial T^{2}}+c_{1} \frac{\partial W_{1}}{\partial T}+F_{1}(t) \frac{\partial^{2} W_{1}}{\partial X^{2}}+\bar{K}\left(W_{1}-W_{2}\right)\right]+E_{1} I_{1} \frac{\partial^{4} W_{1}}{\partial X^{4}}=0, \\
& \mathcal{L}\left[\rho_{2} A_{2} \frac{\partial^{2} W_{2}}{\partial T^{2}}+c_{2} \frac{\partial W_{2}}{\partial T}+F_{2}(t) \frac{\partial^{2} W_{2}}{\partial X^{2}}+\bar{K}\left(W_{2}-W_{1}\right)\right]+E_{2} I_{2} \frac{\partial^{4} W_{2}}{\partial X^{4}}=0,
\end{aligned}
$$


where $\rho_{i}$ and $A_{i}$ are the mass densities and the cross-sectional areas of the beams, $\bar{K}$ is the stiffness of the elastic medium, $W_{i}=W_{i}(X, T)$ are the transverse displacements, $X$ is the axial coordinate, $T$ is the time, $c_{i}$ are the viscous damping coefficients, $E_{i} I_{i}$ are the bending stiffnesses of the beams, and $F_{i}(t)$ are the time-dependent stationary stochastic processes and operator $\mathcal{L}$ is

$$
\mathcal{L}=1-(e a)^{2} \frac{\partial^{2}}{\partial x^{2}}
$$

The boundary conditions for the simply supported edges are

$$
\left.\begin{array}{l}
X=0 \\
X=L
\end{array}\right\} W_{1}=0, \quad \frac{\partial^{2} W_{1}}{\partial X^{2}}=0, \quad W_{2}=0, \quad \frac{\partial^{2} W_{2}}{\partial X^{2}}=0 .
$$

Following the work of Murmu and Adhikari [15], we assume that both nanobeams are identical

$$
\rho_{1} A_{1}=\rho_{2} A_{2}=\rho A, \quad E_{1} I_{1}=E_{2} I_{2}=E I, \quad c_{1}=c_{2}=c .
$$

Now, the system given with expression (1) is non-dimensionalized using the following parameters

$$
\begin{gathered}
T=k t, \quad W_{i}=L w_{i}, \quad X=L x, \quad k_{t}=L^{2} \sqrt{\frac{\rho A}{E I}}, \\
2 \varepsilon \beta=\frac{c k_{t}}{\rho A}, \quad \varepsilon^{I / 2}\left(f_{0 i}+f_{i}(t)\right)=\frac{F_{i}}{\rho A L^{2}}, \quad \varepsilon K=\frac{\bar{K} L^{4}}{\rho A}, \quad(i=1,2),
\end{gathered}
$$

where $\varepsilon$ is a small fluctuation parameter. After applying (5) in (1) the following nondimensional form of Eq. (1) is obtained

$$
\begin{gathered}
\mathcal{L}\left[\frac{\partial^{2} w_{1}}{\partial t^{2}}+2 \varepsilon \beta \frac{\partial w_{1}}{\partial t}+\varepsilon^{1 / 2}\left(f_{01}+f_{1}(t)\right) \frac{\partial^{2} w_{1}}{\partial x^{2}}+\varepsilon K\left(w_{1}-w_{2}\right)\right]+\frac{\partial^{4} w_{1}}{\partial x^{4}}=0, \\
\mathcal{L}\left[\frac{\partial^{2} w_{2}}{\partial t^{2}}+2 \varepsilon \beta \frac{\partial w_{2}}{\partial t}+\varepsilon^{1 / 2}\left(f_{02}+f_{2}(t)\right) \frac{\partial^{2} w_{2}}{\partial x^{2}}+\varepsilon K\left(w_{2}-w_{1}\right)\right]+\frac{\partial^{4} w_{2}}{\partial x^{4}}=0,
\end{gathered}
$$

and

$$
\mathcal{L}=1-\mu^{2} \frac{\partial^{2}}{\partial x^{2}}, \quad \mu=\frac{e a}{L},
$$

where $a$ and $e$ are the characteristic length scale and the nondimensional constant, respectively. 


\section{DisCRETIZATION OF THE EQUATIONS OF MOTION}

Now, by using the Galerkin method system, (6) is reduced to two ordinary differential equations representing only the time varying part of the solution. The first mode of the transverse motion of the beams can be described by

$$
w_{i}(x, t)=q_{i}(t) \sin \pi x, \quad i=1,2 .
$$

By substituting (8) in (6), the following discretizated form of equations (6) is obtained

$$
\begin{gathered}
\ddot{q}_{1}+2 \beta \varepsilon \dot{q}_{1}+\varepsilon K\left(q_{1}-q_{2}\right)+\eta^{2} q_{1}-\varepsilon^{1 / 2} f_{1}(t) \pi^{2} q_{1}=0, \\
\ddot{q}_{2}+2 \beta \varepsilon \dot{q}_{2}+\varepsilon K\left(q_{2}-q_{1}\right)+\eta^{2} q_{2}-\varepsilon^{1 / 2} f_{2}(t) \pi^{2} q_{2}=0,
\end{gathered}
$$

where $\varepsilon \beta$ represents the small viscous damping coefficient, $\varepsilon^{1 / 2} f_{i}(t)$ is the white-noise process with small intensity and

$$
\eta^{2}=\frac{\pi^{4}}{1+\mu^{2} \pi^{2}}
$$

\section{LYAPUNOV EXPONENTS AND STABILITY CONDITIONS}

Basic definitions of stochastic stability are the sample or almost-sure stability and the stability in the mean of the $p$-th order, which is based on the concept of the Lyapunov exponent given in Arnold et al. [16]. The almost-sure stability is described by the maximal Lyapunov exponent defined as

$$
\lambda_{q}=\lim _{t \rightarrow \infty} \frac{1}{t} \ln \left\|q\left(t ; q_{0}\right)\right\|
$$

where $q\left(t ; q_{0}\right)$ is the solution process of a linear dynamic system. It gives the exponential growth rate of the solution. If $\lambda_{q}<0$, then, by definition, $\left\|q\left(t ; q_{0}\right)\right\|^{p} \rightarrow 0$ as $t \rightarrow \infty$, the solution is almost-surely stable, and $\lambda_{q}>0$ implies the instability of the solution in the almost-sure sense. The exponential growth rate, $E\left\|q\left(t ; q_{0}\right)\right\|^{p}$, is provided by the moment Lyapunov exponent defined as

$$
\Lambda_{q}\left(p, q_{0}\right)=\lim _{t \rightarrow \infty} \frac{1}{t} \ln E\left\|q\left(t ; q_{0}\right)\right\|^{p}
$$

where $E$ denotes the expectation. If $\Lambda_{q}\left(p, q_{0}\right)<0$, then by definition, $E\left\|q\left(t ; q_{0}\right)\right\|^{p} \rightarrow \infty$ as $t \rightarrow \infty$, and those conditions are referred to as the $p$-th moment stability. The moment Lyapunov exponent provides us with finer stability properties of the random dynamic system. To have a complete picture of the dynamic stability of a stochastic system it is important to study both the sample and moment stability. 
Following the results from the authors' previous works [9-11], where the regular perturbation method is performed, the analytical expression for the moment Lyapunov exponent is

$$
\Lambda(p)=\varepsilon\left[\frac{p(3 p+10)}{64 \eta^{2}} \pi^{4} \sigma^{2}-p \beta\right],
$$

where $\sigma^{2}$ is the intensity of the white noise process.

Using a property of the moment Lyapunov exponent, the Lyapunov exponent of the double nanobeam system (3) is

$$
\lambda=\left.\frac{d \Lambda(p)}{d p}\right|_{p=0}=\varepsilon\left[\frac{5}{32 \eta^{2}} \pi^{4} \sigma^{2}-\beta\right] .
$$

\section{NUMERICAL RESULTS}

This paper investigates the dynamic stability of two elastically connected nanobeams under white noise excitation. According to the Lyapunov exponent and the moment Lyapunov exponent method, bounds of the almost-sure stability of the observed nanosystem are presented for different system parameters. When the Lyapunov exponent is negative, system (1) is almost-surely stable with probability 1.

Firstly, according to equation (13), numerical results are presented in the plane of the moment Lyapunov exponent $\Lambda(p)$ and the norm degree $p$. Figs. 1 and 2 present the almostsure and $p$-th moment stability boundaries in the function of the damping coefficient $\beta$ and parameter $\varepsilon$, respectively.

Figs. 3 and 4 show the boundaries of the almost-sure stability for $\Lambda(p)=0$. Firstly, in Fig. 3 in the plane of $\sigma^{2}$ and $\beta$ the bounds of the almost-sure stability are given in the function of the norm degree $p$. As shown in the previous figures (Fig. 1 and Fig. 2), this parameter growth leads to the system instability. Similarly, in Fig. 4 the reduction in stability regions is presented for different values of the nanoscale coefficient. This figure is given in the function of the damping coefficient. As already presented in Fig. 1, this parameter growth significantly enlarges stability regions. Thus, a negative influence on the system stability produced by the nonlocal parameter growth can be compensated for higher values of the damping coefficient.

At the end of this study, numerical results of the bounds of the almost-sure stability are given according to the Lyapunov exponent $\lambda$ presented by (14). The results from Fig. 5 are given in the plane of nanoscale coefficient $\mu$ and the intensity of white noise process $\sigma^{2}$, in the function of damping coefficient $\beta$.

As in Fig. 4, where numerical results were obtained according to the moment Lyapunov exponent, it can be seen that an increase in the nanoscale coefficient is followed by a remarkable reduction in stability regions especially for smaller values of the damping coefficient. 


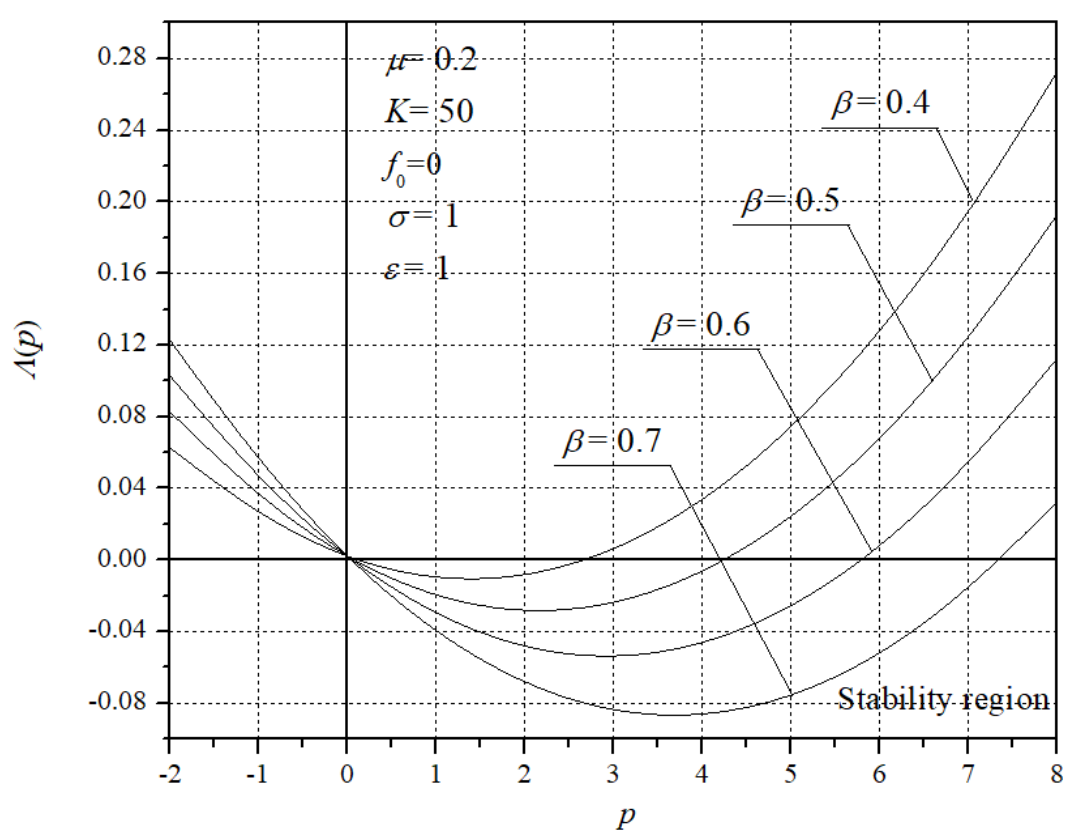

Fig. 1 Stability regions in the function of damping coefficient $\beta$

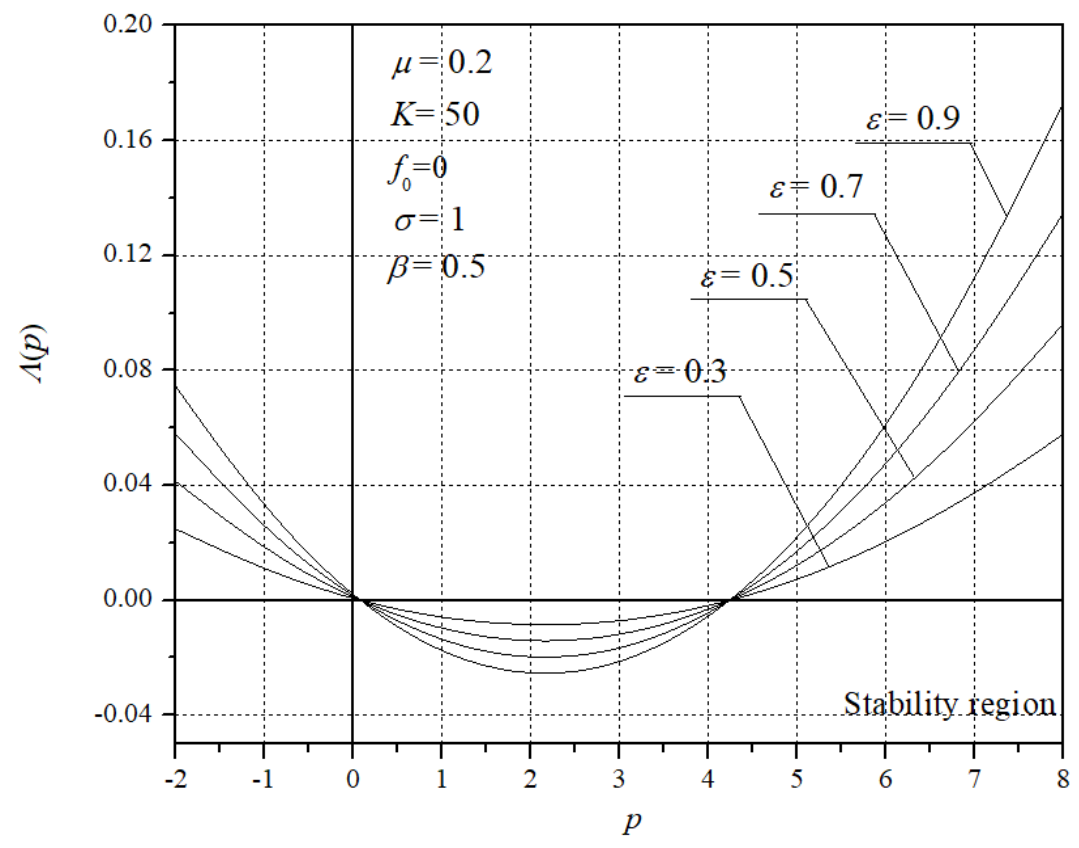

Fig. 2 Stability regions in the function of small fluctuation parameter $\varepsilon$ 


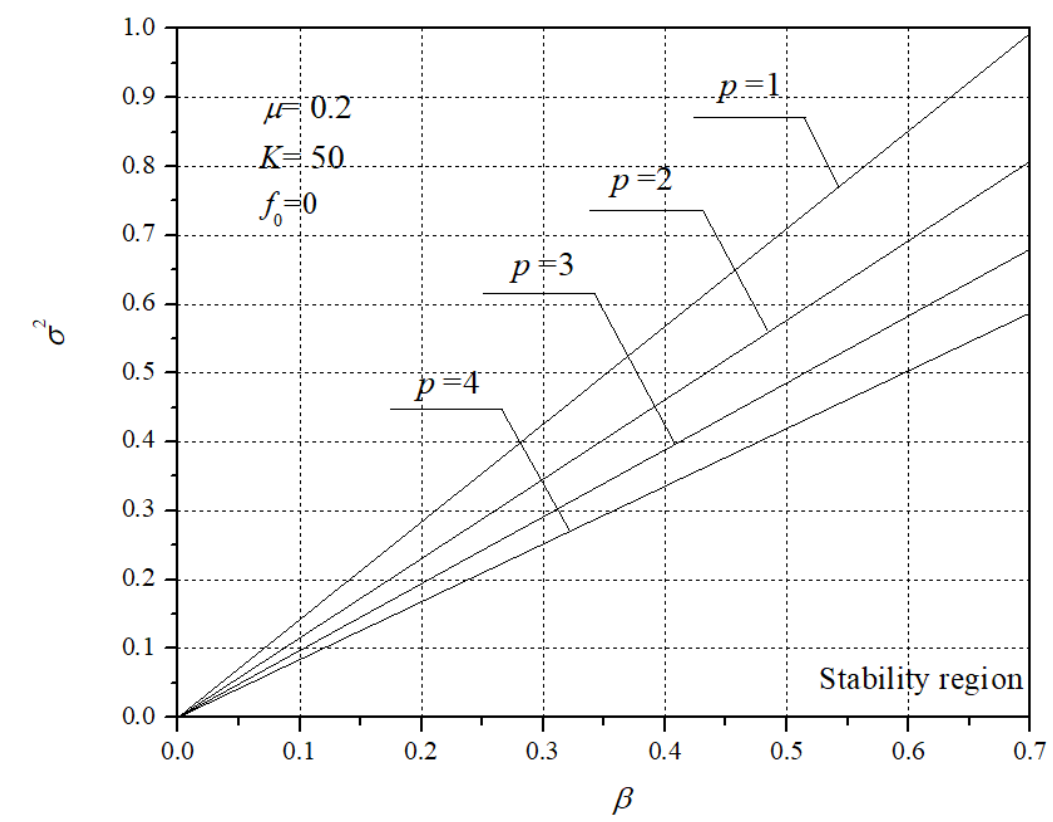

Fig. 3 Stability regions obtained from the moment Lyapunov exponent (14) for $\Lambda(p)=0$ in the function of norm degree $p$

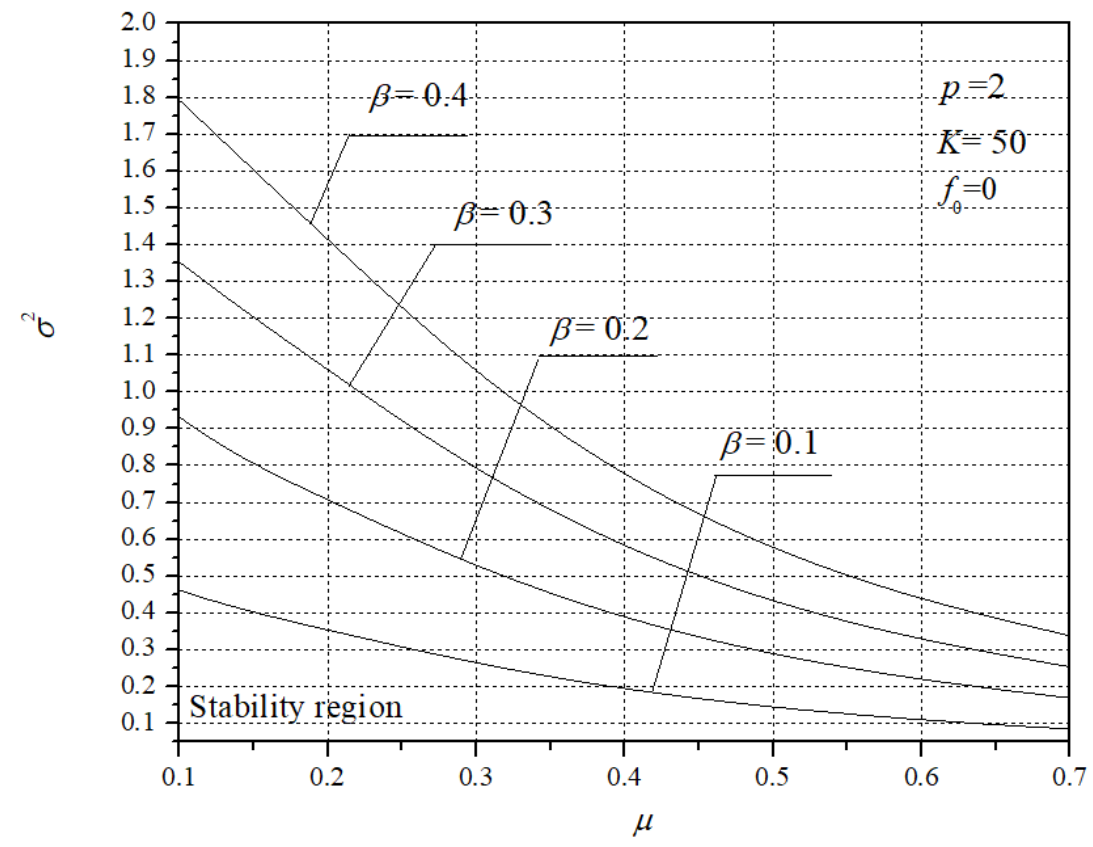

Fig. 4 Stability regions obtained from the moment Lyapunov exponent (14) for $\Lambda(p)=0$ in the function of damping coefficient $\beta$ 


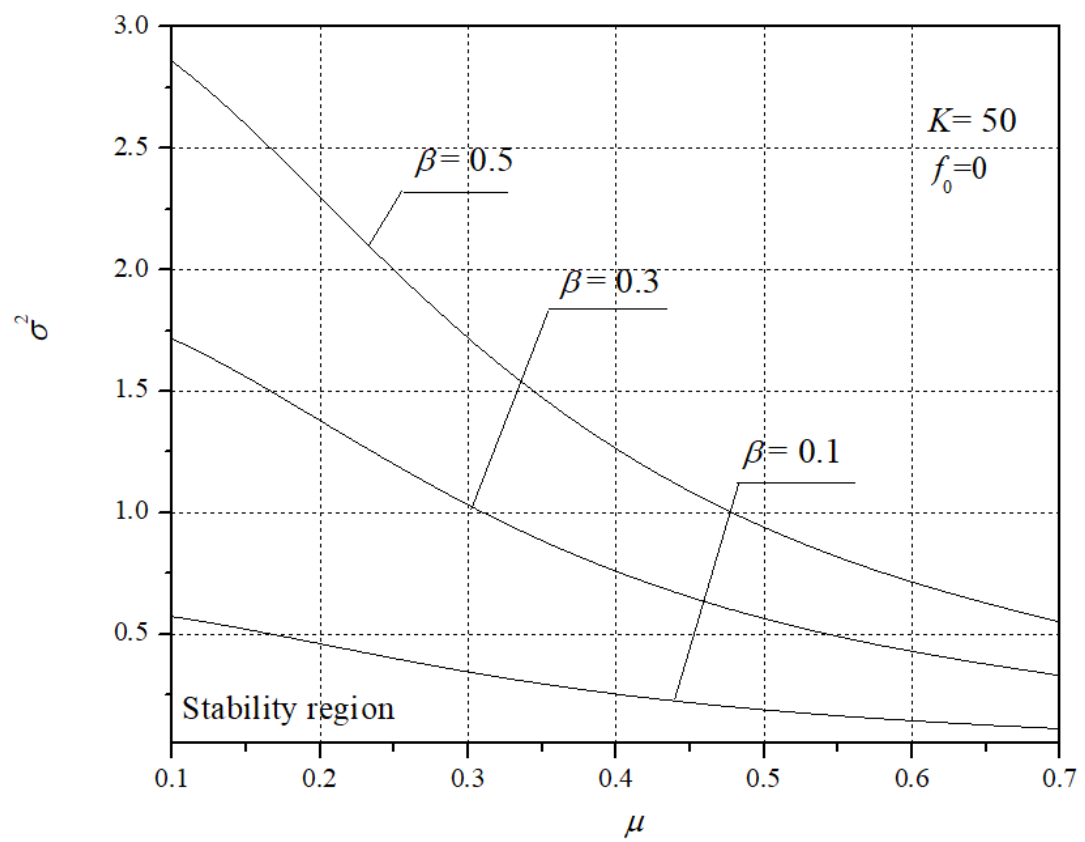

Fig. 5 Stability regions obtained from the Lyapunov exponent result (14) for $\lambda=0$ in the function of damping coefficient $\beta$

\section{CONCLUSION}

By means of the Moment Lyapunov exponent, the stochastic stability of two elastically connected nanobeams under white noise excitation is studied in this paper. The regular perturbation method is applied to obtain analytical results for the moment Lyapunov exponents and Lyapunov exponent in terms of small fluctuation parameter $\varepsilon$. Moment Lyapunov exponents are important characteristic numbers for describing the dynamic stability of a stochastic system. When the $p$-th moment Lyapunov exponent is negative, the $p$-th moment of the solution of the stochastic system is stable.

Regions of almost-sure stability are obtained as a function of the moment Lyapunov exponent $\Lambda$, intensity of white noise process $\sigma^{2}$, damping coefficient $\beta$, norm degree $p$ and nanoscale coefficient $\mu$. As in the authors' previous studies in this field, it is confirmed that nonlocal effects significantly reduce stability regions. The numerical study shows that the negative influence of the nanoscale coefficient on stability regions can be successfully compensated with a growth in the damping coefficient parameter.

Finally, with the aim of comparing the results obtained from the moment Lyapunov exponent with the results obtained from the Lyapunov exponent method, Fig. 4 and Fig. 5 present the influence of the most important parameters for the system stability, $\beta$ and $\mu$.

Acknowledgements: This paper was supported by the Ministry of Education and Science of the Republic of Serbia, through the project No 174011. 


\section{REFERENCES}

1. Ansari, R., Torabi, J., Norouzzadeh, A., 2018, Bending analysis of embedded nanoplates based on the integral formulation of Eringen's nonlocal theory using the finite element method, Physica B: Condensed Matter, 534, pp. 90-97.

2. Zhang, H., Wang, C.M., Challamel, N., Pan, W.H., 2020, Calibration of Eringen's small length scale coefficient for buckling circular and annular plates via Hencky bar-net model, Applied Mathematical Modelling, 78, pp. 399-417.

3. Pavlović, I., Pavlović, R., Janevski, G., 2016, Dynamic instability of coupled nanobeam systems. Meccanica, 51, pp. 1167-1180.

4. Huang, Y., Fu, J., Liu. A., 2019, Dynamic instability of Euler-Bernoulli nanobeams subject to parametric excitation, Composites Part B: Engineering, 164, pp. 226-234.

5. Jena, S.K., Chakraverty, S., 2018, Free vibration analysis of Euler-Bernoulli nanobeam using differential transform method, International Journal of Computational Materials Science and Engineering, 7(3), pp. 1850020.

6. Zhang, Y.Q., Lu, Y., Ma, G.W., 2008, Effect of compressive axial load on forced transverse vibration of a double-beam system. International Journal of Mechanical Sciences, 50(2), pp. 299-305.

7. Xie, W.-C., 2003, Moment Lyapunov exponents of a two-dimensional system under bounded noise parametric excitation. Journal of Sound and Vibration 263(3), pp. 593-616.

8. Xie, W.-C., 2001, Moment Lyapunov exponents of a two-dimensional system under real-noise excitation. Journal of Sound and Vibration 239(1), pp. 139-155.

9. Kozić, P., Janevski, G., Pavlović, R., 2010, Moment Lyapunov exponents and stochastic stability of a double-beam system under compressive axial loading, International Journal of Solids and Structures, 47(10), pp. 1435-1442.

10. Kozić, P., Pavlović, R., Janevski, G., 2008, Moment Lyapunov exponents of the stochastic parametrical Hill's equation, International Journal of Solid and Structures, 45(24), pp. 6056-6066.

11. Pavlović, R.I., Karličić, Z.D., Pavlović, R., Janevski, B.G., Ćirić, T.I., 2016, Stochastic stability of multi-nanobeam systems, International Journal of Engineering Science, 109, pp. 88-105.

12. Pavlović, I., Pavlović, R., Kozić, P., Janevski, G., 2013, Almost sure stochastic stability of a viscoelastic doublebeam system, Archive of Applied Mechanics, 83, pp. 1591-1605.

13. Ru, C.Q., 2000, Effect of van der Waals forces on axial buckling of a double-walled carbon nanotube, Journal of Applied Physics, 87(10), pp. 7227-7231.

14. Eringen, A.C., 2002, Nonlocal Continuum Field Theories, Springer-Verlag, New York.

15. Murmu, T., Adhikari, S., 2011, Axial instability of double nanobeam-systems, Physics Letters A, 375(3), pp. 601-608.

16. Arnold, L., Doyle, M.M., Sri Namachchivaya, N., 1997, Small noise expansion of moment Lyapunov exponents for two-dimensional systems, Dynamics and Stability of Systems, 12(3), pp. 187-211. 